\title{
The PIERS Experience: Research or Quality Improvement?
}

\author{
Tabassum Firoz, MD, FRCPC, ${ }^{1}$ Laura A. Magee, MD, FRCPC, MSc, FACP, ${ }^{1,2,3,4}$ \\ Beth A. Payne, BSc, ${ }^{2,3}$ Jennifer M. Menzies, MSc, ${ }^{2,3}$ Peter von Dadelszen, MBChB, DPhil, FRCSC ${ }^{2,3,4}$; \\ for the Pre-eclampsia Integrated Estimate of RiSk (PIERS) Study Group
}

${ }^{1}$ Department of Medicine, University of British Columbia, Vancouver BC

${ }^{2}$ Department of Obstetrics and Gynaecology, University of British Columbia, Vancouver BC

${ }^{3}$ Child and Family Research Institute, University of British Columbia, Vancouver BC

${ }^{4}$ School of Population and Public Health, University of British Columbia, Vancouver BC

\section{INTRODUCTION}

$\mathrm{T}$ The PIERS project was run as a prospective multicentre international study in seven centres in Canada, the United Kingdom, New Zealand, and Australia. Standardized assessment, by means of pre-printed orders for maternal and fetal surveillance, was used to document information about potential predictors of a composite adverse maternal outcome developed by content experts through Delphi consensus. ${ }^{1}$ Standardization of assessment has been shown to improve outcomes for a variety of conditions such as pulmonary embolism. ${ }^{2}$

The fullPIERS clinical prediction model was developed and validated for women with preeclampsia who were admitted to tertiary care hospitals. ${ }^{3}$ The inclusion and exclusion criteria are detailed elsewhere. ${ }^{3}$ The fullPIERS model identified the following predictors of adverse maternal outcome within 48 hours of admission: gestational age, chest pain or dyspnea, oxygen saturation, platelet count, serum creatinine, and serum aspartate transaminase concentration (for this model, the area under the curve of the receiver operating characteristic was $0.88,95 \%$ CI 0.84

J Obstet Gynaecol Can 2012;34(4):379-381

Key Words: Quality improvement, consent, ethics boards, pregnancy research

Competing Interests: None declared.

Received on September 20, 2011

Accepted on November 22, 2011 to 0.92$){ }^{4}$ There were, however, many other potential predictors considered, and many of these clinical and laboratory measures are of particular interest to clinicians. Univariable analyses related to these measures have also been discussed in a number of separate publications. ${ }^{5,6}$

What may also be of interest to clinicians, beyond the content of PIERS, is the use in PIERS of standardization of clinical practice, and in particular, the experience of running the project as a continuous quality improvement project in some centres, while informed consent was required in others. In this commentary, we describe our experience and outline the implications of these two approaches for patient recruitment, cost, and resource use.

\section{CONTINUOUS QUALITY IMPROVEMENT AS RESEARCH}

The divide between CQI and research has not always been clear. Historically, CQI was not considered research as it was a process of reaction to bad outcomes. ${ }^{7}$ However, with improved study design and statistical methods, it is no longer considered a separate entity from research.

CQI provides infrastructure for conducting clinical and outcomes research and promotes evaluation in clinical practice. $^{8}$ CQI projects use existing data (from medical records, databases, or registries) to learn from what is being done in clinical practice. The strength of this approach lies in the ability to include all patients with a given condition, or at least a representative sample of them. In contrast, requiring informed consent from individual patients introduces sampling bias into the type of individual 
enrolled, particularly when consent rates are low. This compromises the scientific validity of the data and may bias participation towards those with less severe disease. Inter-hospital variability in consent rates may further magnify the bias and make its impact on outcomes more difficult to understand.

\section{THE PIERS EXPERIENCE}

Initially, PIERS was run in three centres ( $\mathrm{n}=943$ women) as a CQI project and in four centres $(\mathrm{n}=316$ women) as a study requiring informed consent from women for secondary analysis of existing data from their medical records. The choice of methodology was dictated by the local research ethics board. Although the 2010 Tri-Council Policy Statement does not require researchers to seek consent from individuals for the secondary use of non-identifiable information (as in PIERS), research ethics boards in some PIERS centres in Canada required informed consent for study approval.

\section{Consent Rates}

In PIERS, centres that were required to obtain informed consent for data collection had consent rates of $29 \%$, $83 \%, 91 \%$, and $92 \%$. Coordinators gave many reasons for patients refusing to give informed consent and many of those reasons were not surprising, such as lack of patient interest or inadequate English or French skills to understand the study explanation; however, an important reason for refusal of consent was severe (as opposed to non-severe) preeclampsia, which is associated with the highest rates of adverse outcomes. A critical number of adverse outcomes are required to build an outcome prediction model, particularly when a large number of candidate predictors are considered.

As PIERS could not collect information on women who did not consent to PIERS data collection in non-CQI sites, we cannot comment on differences in outcomes related to informed consent. However, in the initial PIERS site, more adverse outcomes $(5.1 \%)$ were seen when the study moved from obtaining informed consent to use standardized pre-printed orders for maternal and fetal assessment in preeclampsia than in the epoch when PIERS was run as a CQI project $(0.7 \%)$ and all women admitted to hospital with preeclampsia underwent standardized assessment according to pre-printed orders ( $95 \%$ CI 0.04 to $0.49 .{ }^{10}$

\section{ABBREVIATIONS}

CQI continuous quality improvement

PIERS Pre-eclampsia Integrated Estimate of RiSk
The transition to CQI also resulted in the pre-printed orders being used consistently for $92 \%$ of women admitted with preeclampsia, compared with the previous epoch in which these orders were used for only $42 \%$ of women who had given their informed consent for participation in PIERS.

\section{Resources and Costs}

Staffing also played a critical role in recruitment in nonCQI PIERS centres. In one such centre, 66 women met PIERS eligibility criteria but were not recruited because of lack of staff to obtain consent from patients, particularly overnight and on weekends.

The PIERS research costs were higher in non-CQI sites. In both CQI and non-CQI sites, approximately seven hours were required for PIERS case identification, data retrieval from case files, data entry into the PIERS database, and resolution of data queries from the central PIERS site. However, sites that were required to obtain informed consent for PIERS data collection required an additional 1.25 hours of coordinator time, particularly as the consent process often took two visits. This additional time translates into higher costs, although a formal cost analysis was not performed.

\section{PIERS in Relation to the Medical Literature}

We were unable to identify pregnancy studies examining the impact on consent rates or outcomes of informed consent for secondary data analysis. However, observations in studies outside pregnancy were consistent with the observations that we made during the conduct of PIERS. A systematic review of 17 non-pregnancy studies identified highly variable published consent rates of $37 \%$ to $93 \%$ for data abstraction from existing medical records. ${ }^{11}$ These often low and variable rates of consent were also seen in the Canadian Acute Stroke Registry when it was forced to transition from anonymous record review to informed consent; the consent rate was $51 \%$ even after a year's experience in recruitment methods, and the inter-site variation in consent was $17 \% .{ }^{12,13}$ This registry also identified the need for multiple visits to introduce the study, explain the procedures, and give ample opportunity to ask questions and make a decision about participation. ${ }^{12}$ The bias in patients recruited was also illustrated by the significantly lower in-hospital mortality rate (6.9\%) among patients who consented to registry participation, as opposed to those who did not $(21.7 \%) .{ }^{12}$

\section{A BROADER CONCEPT OF CONSENT}

A recent commentary by Hansson argues for a broader understanding of autonomy and informed consent. ${ }^{14}$ It argues that having a restricted view of autonomy 
undermines the possibility of patients participating in the development of medical science. The author states that even if there is limited risk of harm (such as in a database/registry study), research ethics boards focus on the individual's interest in having a say. However, this is at the expense of a drop-out rate that may result in the inability to draw scientifically meaningful conclusions from the study. Instead, Hansson proposes an expanded view of autonomy in which there is no consent, but the individual has a right to know what type of information is in the registry and has access to indirect means of exercising insight and influencing decision-making,

\section{CONCLUSION}

There are lessons to be learned from PIERS in terms of both content and methods. The standardized assessment of hospitalized women with preeclampsia in PIERS yielded great insights by standardizing what we do in routine clinical practice and auditing the results. However, in PIERS we saw research and financial challenges associated with requirements for informed consent for secondary analysis of existing data that were similar to those seen in studies outside pregnancy. We urge research ethics boards and institutional review boards to consider carefully the impact of requiring such an approach on research quality and costs, particularly as the Tri-Council policy does not require such an approach to protect an individual's privacy.

\section{ACKNOWLEDGEMENTS}

We would like to thank the PIERS site co-ordinators (Lynn Bissonnette, Sherbrooke, QC; Laura Payant and Svetlana Shachkina, Ottawa, ON; Heather Ramshaw, Kingston, ON; Jane Hayes, Leeds, UK; Barbra Pullar, Christchurch, New Zealand; and Claire Parker, Subiaco, Australia).

\section{REFERENCES}

1. Linstone HA, Turoff M. The Delphi Method: techniques and applications. Reading, MA: Adison-Wesley; 1975. ISBN 9780201042948. Available at: http://is.njit.edu/pubs/delphibook. Accessed January 11, 2012.

2. Wells PS, Ginsberg JS, Anderson DR, Kearon C, Gent M, Turpie AG, et al. Use of a clinical model for safe management of patients with suspected pulmonary embolism. Ann Intern Med 1998;129: 997-1005.

3. von Dadelszen P, Payne B, Li J, Ansermino JM, Broughton Pipkin F, Douglas MJ, et al.; for the PIERS Study Group. Prediction of adverse maternal outcomes in pre-eclampsia: development and validation of the fullPIERS model. Lancet 2011;377(9761):219-27. Comment in Lancet 2011;377(9761):185-6.

4. PIERS Calculator. Available at: http://piers.cfri.ca/PIERSCalculatorH.aspx. Accessed August 23, 2011.

5. Payne B, Magee LA, Cote Anne-Marie, Hutcheon JA, Li Ji, Kyle PM, et al. PIERS proteinuria: relationship with adverse maternal and perinatal outcome. J Obstet Gynaecol Can 2011;33:588-97.

6. Millman AL, Payne B, Qu Ziguang, Douglas J, Hutcheon JA, Lee T, et al. Oxygen saturation as a predictor of adverse maternal outcomes in women with pre-eclampsia. J Obstet Gynaecol Can 2011; 33:705-14.

7. American College of Surgeons. Continuous quality improvement. Available at: http://www.facs.org/cqi/index.html. Accessed October 17, 2011.

8. Bellin E, Dubler NN. The quality improvement-research divide and the need for external oversight. Am J Public Health 2001;91:1512-7.

9. Canadian Institutes of Health Research, Natural Sciences and Engineering Research Council of Canada, Social Sciences and Humanities Research Council of Canada. Tri-Council policy statement: ethical conduct for involving humans, 2010. Available at: http://www.pre.ethics.gc.ca/pdf/ eng/tcps2/TCPS_2_FINAL_Web.pdf. Accessed July 28, 2011.

10. Menzies J, Magee LA, Li J, Macnab YC, Yin R, Stuart H, et al. Instituting surveillance guidelines and adverse outcomes in preeclampsia. Obstet Gynecol 2007;110:121-7.

11. Kho ME, Duffet M, Willison DJ, Cook DJ, Brouwers MC. Written informed consent and selection bias in observational studies using medical records: systematic review. BMJ 2009;338:b866.

12. Tu JV, Willison DJ, Silver FL, Fang J, Richards JA, Laupacis A, et al. Impracticability of informed consent in the Registry of the Canadian Stroke Network. N Engl J Med 2004;350:1414-21.

13. Willison DJ, Kapral MK, Peladeau P, Richards JA, Fang J, Silver FL. Variation in recruitment across sites in a consent-based clinical data registry: 1essons from the Canadian Stroke Network. BMC Med Ethics 2006;7:1-8.

14. Hansson M. Do we need a wider view of autonomy in epidemiological research? BMJ 2010;340:1172-4. 